\title{
Qualitative analysis of Sudan IV in edible palm oil
}

Sampson Saj Andoh ${ }^{1^{*}}$ (D, Tarmo Nuutinen ${ }^{2}$, Cheetham Mingle ${ }^{3}$ and Matthieu Roussey ${ }^{1}$

\begin{abstract}
Background: Palm oil is one of the most useful vegetable available. Sudan IV dye is used as hue enhancer in palm oil despite the ban as food colorant due to its carcinogenicity and mutagenicity by the International Agency for Research on Cancer (IARC).

Methods: Surface enhanced Raman spectroscopy (SERS) coupled with chemometric methods was applied to detect the presence of Sudan IV in some edible palm oil samples.

Results: We studied the samples within the 1200-1800 $\mathrm{cm}^{-1}$ Raman frequency range. In predicting adulteration, we used $1388 \mathrm{~cm}^{-1}$ Raman peak that is associated with Sudan IV as our marker. We were able to confirm adulteration in four of the five palm oil samples provided by the Food and Drug Authority of Ghana.

Conclusions: With these methods, we confirmed the results from Food and Drug Authorities of Ghana by proving that there were indeed Sudan IV adulteration in some palm oil samples.
\end{abstract}

Keywords: Sudan IV, Palm oil, Adulteration, SERS, And authentication

\section{Introduction}

Palm oil is a bright reddish/orange oil produced from the mesocarp of the fruits of the oil palm trees. They are very nutritious and has many uses around the world. In some communities, they are used for treating some ailment such as vitamin A deficiency [1]. Palm oil is currently the most used oil in the food industry, as it is present in almost all ready-to-eat foods [2]. This market is encouraged by the palm oil's low cost, and its convenient usage, i.e., its ability to easily decolorize and semi solid nature at temperatures lower than $25^{\circ} \mathrm{C}$. Palm oil from West African countries such as Nigeria and Ghana is considered as one of the best available due to the higher levels of $\beta$-carotene in them. Edible palm oil is a great source of foreign exchange for Ghana by serving as a major non-traditional export. In recent times, due to the rise in utilization of palm oil especially in ready-to-eat meals, it has become a preferential target for counterfeiting and adulteration. The use of Sudan IV as an adulterant to improve the hue of palm oil is unfortunately on the rise. In one point in time, Sudan IV

\footnotetext{
* Correspondence: sampson.andoh@uef.fi

${ }^{1}$ Institute of Photonics, University of Eastern Finland, 80101 Joensuu, Finland Full list of author information is available at the end of the article
}

adulteration was considered the second most Sudan dye adulterant for food [3].

Sudan IV dye is a fat-soluble azo-dye used as an industrial colorant for tiles, polishes, and other industrial products [4]. Because of the demonstrated significant carcinogenic and mutagenic properties of these dyes, the International Agency for Research on Cancer (IARC) has banned them as food additives in all forms and quantity as they are no known tolerable daily intakes [58]. Because of their low cost, bright colors, wide availability, and easy accessibility [6], some dishonest people employ such Sudan IV dye as palm oil hue enhancer. As in all complex liquids, it is difficult to detect Sudan IV adulteration in palm oil. However, palm oil adulteration becomes a public health, consumer confidence $[2,8]$ and a global issue, yielding more attention in developing methods for authenticating palm oil and detecting such Sudan IV adulterations.

Different methods already exist to authenticate and detect adulteration in edible palm oil. Prominent in these methods are high-performance liquid chromatography (HPLC), liquid chromatography-mass spectrometry (LC-MS)/mass spectrometry (MS), mid-infrared spectroscopy (MIR), and Fourier transform infrared 

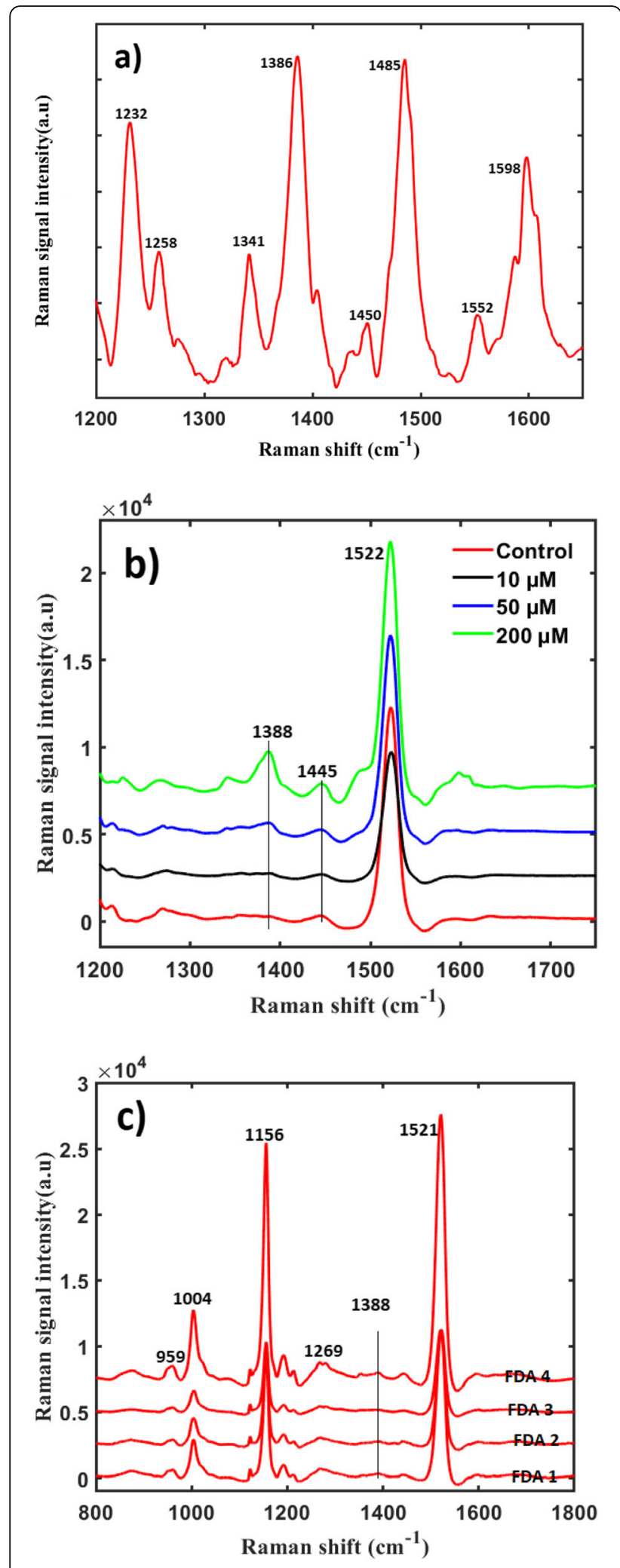

Fig. 1 SERS spectra of a) $1 \mu \mathrm{M}$ Sudan IV dye b) the unadulterated and the prepared palm oil samples with different concentrations of Sudan IV and $\mathbf{c}$ ) the other palm oil samples obtained from FDA Ghana. The measurements were conducted at $\lambda=514 \mathrm{~nm}$ with a lasing power of $50 \mu \mathrm{W}$ and an acquisition time $10 \mathrm{~s}$

spectroscopy (FTIR) [2, 9-11]. These methods produce highly accurate, most efficient and repeatable results. They however possess serious setbacks, which include requiring a high level of expertise in operation, time consumption, and other factors such as an extensive sample preparation [11]. Other vibrational spectroscopic methods such as surface enhanced Raman spectroscopy (SERS), which has a potential to overcome such drawbacks, may be employed. SERS is a rapid and sensitive vibrational spectroscopic technique based on Raman scattering [12].

In this work, we report on the use of surface enhanced Raman spectroscopy in authenticating and detecting adulteration in edible palm oil from Ghana. We applied principal component analysis (PCA), a statistical analysis tool, on the SERS spectra to produce data that complements the results obtained in SERS. PCA is a wellknown technique of data representation used for analyzing complex and heavy datasets [13] based on multivariate analysis. PCA separates data in orthogonal variables that makes it easier to analyze [14]. By mapping in a two-dimensional plan, principal component analysis allows a deeper, although qualitative, discrimination between authentic and adulterated palm oil samples.

\section{Results and discussion}

Surface-enhanced Raman spectroscopy

A set of samples comprising of, in-laboratory prepared and collected in Ghana palm oil samples (see methods), were measured by SERS. The SERS profiles are key tools enabling the authentication and subsequent detection of adulteration in palm oil. In Fig. 1 below, the SERS spectra of Sudan IV and all the oil samples is presented within the frequency range $1200-1800 \mathrm{~cm}^{-1}$.

As expected, we observed bands and peaks in the SERS spectra of Sudan IV including 1232, 1258, 1386, and $1598 \mathrm{~cm}^{-1}$, see Fig. 1a. These are assigned to the $\gamma\left(\mathrm{C}=\mathrm{CH}_{3}\right)$ stretching, $\delta(\mathrm{C}-\mathrm{H})$ deformation, $\mathrm{CH}_{3}$ band, amine I protein of the Sudan IV molecule, respectively. There are also a $\mathrm{C}-\mathrm{H}$ bending at $1485 \mathrm{~cm}^{-1}$ and amine II stretching at $1552 \mathrm{~cm}^{-1}$. In this work, we used the $\mathrm{CH}_{3}$ band at $1386 \mathrm{~cm}^{-1}$ in our analysis for adulteration of the palm oil samples. One can remark, from Fig. 1b, that the amplitude of the peak (at $1386 \mathrm{~cm}^{-1}$ ) increases with the concentration of the adulterant. Note a slight shift in the $1386 \mathrm{~cm}^{-1}$ peak to $1388 \mathrm{~cm}^{-1}$ in all the oil samples. This could be attributed to the influence of other components in the palm oil samples. However, a 
characteristic Raman scattering wavenumber is common to all the oil samples. This is the strong resonance around $1522 \mathrm{~cm}^{-1}$ (Fig. 1b) which is assigned as the $v_{1}(\mathrm{C}=\mathrm{C})$ vibrations, of the $\beta$-carotene molecule [16], which is abundant in palm oil. Other bands and peaks such as the 1303 and $1449 \mathrm{~cm}^{-1}$, observed in the spectra of the oil samples, originate exclusively from the main constituents of the palm oil. These scissoring, deformation, vibrations, and stretching of the palmitic (C16), oleic $(\mathrm{C} 18: 1)$ and linoleic acid (C18:2) produce these bands and peaks in the measured spectra. Considering only the $1386 \mathrm{~cm}^{-1}$ peak, it can be observed from Fig. 1c that there is a slight weak peak in all the FDA samples. This confirms our prior knowledge of adulteration indicated by the FDA-Ghana.

\section{Principal components analysis}

Principal component analysis was used on the sample sets to discriminate between the adulterated and pure palm oil samples. The PCA is performed on the frequency range surrounding the peaks at $1388 \mathrm{~cm}^{-1}$ that are characteristics of Sudan IV. Selecting this range allows to avoid the prominent peak due to $\beta$-carotene at $1521 \mathrm{~cm}^{-1}$. Results are presented in Fig. 2. It is important to note that with the SERS measurements are done following the same procedure and under the same conditions for all the samples. However, the absolute amplitude of the peaks is not always proportional or related to concentration, because some chemical activity may occur when Sudan IV dye is mixed with palm oil. PCA results is an efficient tool to discriminate the adulterated samples but does not provide a quantitative value. Moreover, PCA is relating all the tiny changes in the spectra, including ones coming from the chemical activity. One can observe for instance the drift to the right of the prepared samples as a function of the concentration. This is because these samples were prepared by mixing the acetone/Sudan IV solution to the palm oil. An excess of acetone leads to an enhanced chemical activity modifying almost completely the PCA result. The prepared sample with a $200 \mu \mathrm{M}$ concentration should not be taken into account. To analyze the results provided by the PCA technique, one must consider (in our case) three parameters: 1) the distance to the abscise axis, 2) the quadrant, and 3) the distance to the control point abscise.

As an example (see Fig. 2), the FDA samples yield points with a nearly same abscise than the control point. It means nearly no chemical activity. More the distance from the origin is short, more the sample is possibly adulterated at the condition that the point is in another quadrant than the control point.

From Fig. 2, one can clearly see the very well separated control solution labelled as unaltered by the Food and

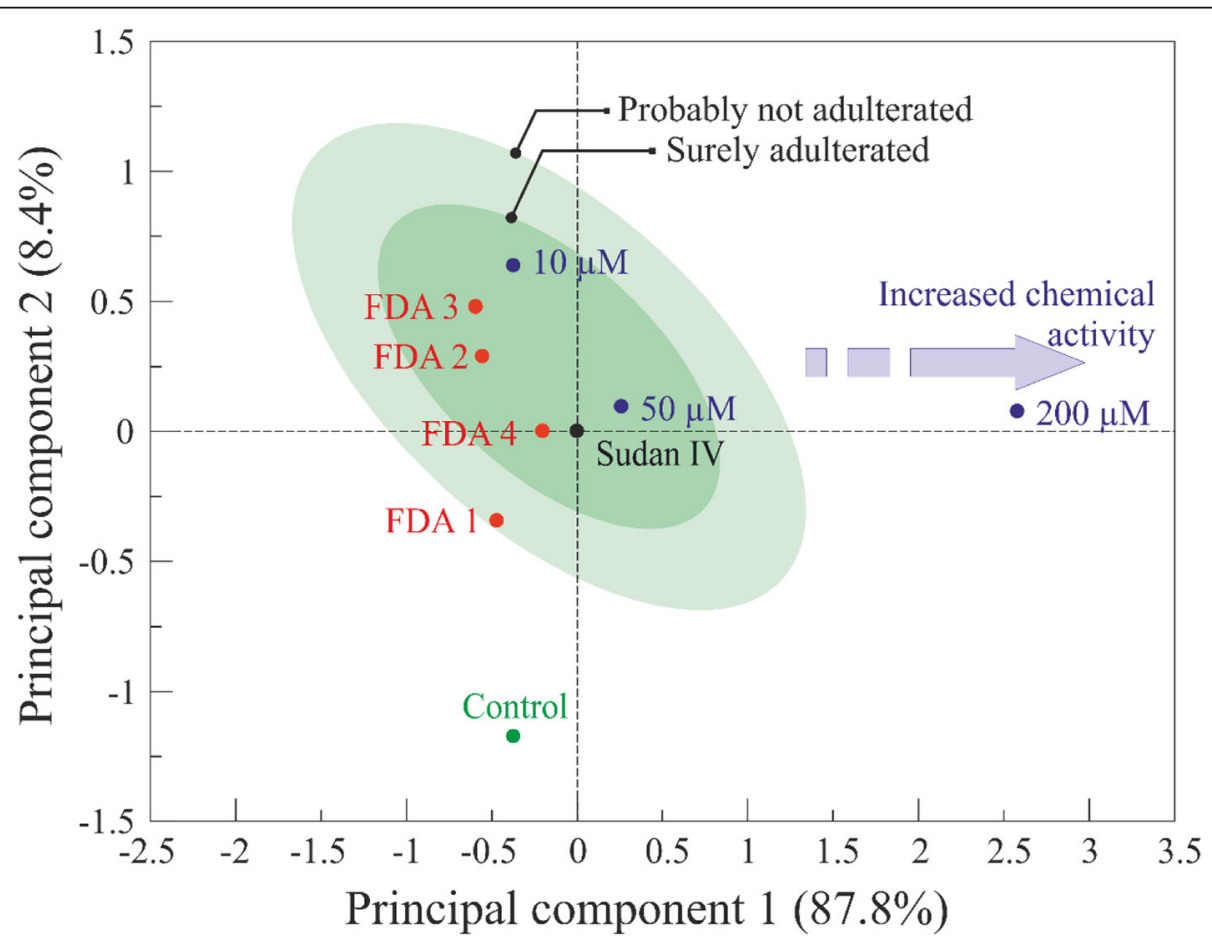

Fig. 2 Principal component analysis performed on the prepared (blue), FDA collected (red), and pure (green) palm oil samples as well as on the Sudan IV solution (black). The dark ellipse represents the area for which samples are surely adulterated. The light green ellipse corresponds to palm oil samples probably not adulterated 
Drug Authority of Ghana. The three solutions prepared in laboratory with a known concentration are used to determine a referential establishing the different zones of adulteration or not. The three of them are in other quadrants than the control point, which means they are corresponding to adulterated samples, as expected. Sudan IV is taken as a referential point also and is centered at the origin of the graph. FDA samples 2, 3, and 4 are also in another quadrant than the control point. They can be considered as adulterated. The FDA 1 sample has a response in the same quadrant than the control point, which means it is probably a non-adulterated palm oil.

It is to be noted than many factors affect the PCA results. The fabrication technique of palm oil may change the result. In other words, another adulterant other than Sudan IV may be present in the FDA 1 sample. One must finally remark that the results also depend on the spectral range taken into account.

\section{Conclusion}

The FDA-Ghana, through the Food Physio-Chemical laboratories unit, provided the test samples used in this study. Ghana accounts for less than $0.1 \%$ of global production of palm oil and yet, Ghana is considered one of the worst culprits when considering Sudan IV dye adulteration of palm oil. By measuring and analyzing the SERS spectra of Sudan IV, prepared palm oil and other palm oil samples, we were able to detect traces of Sudan IV dyes in all four of the palm oil samples provided for analysis. This confirms the prior knowledge of adulteration in them. This method also eliminates sample preparation that is consistent with most high performing analytical tools such as HPLC and others. However, PCA results gave additional inputs on one sample that may not be adulterated, which proves the importance of deeper analysis. The results of this study demonstrated the capabilities of SERS combined with PCA to authenticate and detect adulteration of edible palm oil with Sudan IV dye.

\section{Methods}

\section{Chemical reagents and samples}

All reagents used were of analytical grade. Sudan IV, silver nitrate and sodium chloride were purchased from Sigma-Aldrich (St. Louis, Mo., U.S.A. Putity $>99.99 \%$ ). A $1 \mu \mathrm{M}$ stock solution of Sudan IV was prepared in acetone and used in spiking the palm oil to make the in-laboratory prepared samples for measurements.

Five edible palm oil samples were also obtained from the Food and Drugs Authority (FDA), Ghana. FDAGhana is the central competent authority in charge of food and drug safety in Ghana. The samples were all indicated adulterated with Sudan IV (labelled FDA 1 to 4 )
Table 1 A table showing the prepared palm oil samples with the volume of unadulterated palm oil and Sudan IV dye to obtain the required concentrations required

\begin{tabular}{lll}
\hline Volume $[\mu \mathrm{L}]$ & & Sudan IV concentration $[\mu \mathrm{M}]$ \\
\hline Sample K & Sudan IV & \\
\hline 800 & 200 & 200 \\
950 & 50 & 50 \\
990 & 10 & 10 \\
\hline
\end{tabular}

except one (labelled control/sample K). Palm oil is semisolid at temperatures below $25^{\circ} \mathrm{C}$, therefore the samples were measured on a modified Raman microscope sample stage allowing a temperature control of the sample. The temperature was kept at $45 \pm 2{ }^{\circ} \mathrm{C}$ during the entire measurement. In addition to the samples from FDA, and the Sudan IV, three other samples were prepared by vortexing until complete homogeneity different amount of Sudan IV in unadulterated palm oil. Table 1 shows the quantities of both Sudan IV and palm oil in preparing the required concentrations.

\section{Substrate fabrication}

A silver-chloride nanoparticulate substrate ( $\mathrm{AgCl} \mathrm{NPs}$ ) was prepared according to ref. [15]. Shortly, $\mathrm{AgCl}$ NPs were grown on a circular singly polished side silicon wafer (purchased from Smat) by repeatedly and periodically immersing the wafer in two beakers containing 5 $\mathrm{mM}$ precursor solutions of silver nitrate $\left(\mathrm{AgNO}_{3}\right)$ and sodium chloride $(\mathrm{NaCl})$ with 50 cycles of immersions. The $\mathrm{AgCl}$ nanoparticles are produced as intermediary step to prevent the tarnishing of the silver particles. The synthesis was carried out in ambient laboratory conditions. Then metallic Ag NPs are obtained by the photochemical reaction: $2 \mathrm{AgCl}(\mathrm{s})+h v \rightarrow 2 \mathrm{Ag}(\mathrm{s})+\mathrm{Cl}_{2}(\mathrm{~g})$.

\section{Raman analyses}

A Renishaw inVia Raman microscope was used for all the SERS measurements. A modification was made to the sample stage by mounting a heating system to heat and keep the palm oil samples in the liquid form throughout the measurement. An excitation wavelength of $514 \mathrm{~nm}$ and a laser power of $50 \mu \mathrm{W}$ were utilized. All data were collected with an integration time of $10 \mathrm{~s}$. The readings between $1200 \mathrm{~cm}^{-1}$ to $1800 \mathrm{~cm}^{-1}$ were subsequently analyzed.

Principal components analysis was applied to the SERS spectra to generate a graphical plot of the score. This was used in establishing the authenticity of the palm oil samples from FDA-Ghana against known adulterated samples. The PCA was done using OriginPro 2019 software. 


\section{Abbreviations}

IARC: International Agency for Research on Cancer; PCA: principal component analysis; SERS: Surface-enhanced Raman spectroscopy

\section{Acknowledgements}

The authors would like to thank Tommi Itkonen for his technical support.

\section{Authors' contributions}

SSA and TN conceived, designed, initiated and conducted the measurements of the research. MR analyzed the data by PCA and supervised the whole work. CM supervised and gave access to the FDA samples. All authors contributed to writing of the final manuscript.

\section{Funding}

The study was carried out under the project ChemiReader funded by Business Finland (No 2387/31/2017, collection of the samples in Ghana) and the Academy of Finland project (No SA-298298, salary for TN).

\section{Availability of data and materials}

Detail about data has been provided in the manuscript.

\section{Ethics approval and consent to participate}

Not applicable.

\section{Consent for publication}

Not applicable.

\section{Competing interests}

The authors declare no competing interests.

\section{Author details}

IInstitute of Photonics, University of Eastern Finland, 80101 Joensuu, Finland. ${ }^{2}$ Institute of Photonics and Department of Environmental and Biological Sciences, University of Eastern Finland, P. O. Box 111, Fl-80101 Joensuu, Finland. ${ }^{3}$ Food Physio-Chemical laboratories, Food and Drugs Authority, P. O. Box CT 2783, Cantonments Accra, Ghana.

Received: 1 March 2019 Accepted: 6 September 2019

Published online: 11 September 2019

\section{References}

1. Narasinga, R.B.S.: Potential use of red palm oil in combating vitamin a deficiency in India. Food Nutr. Bull. 21(2), 202-211 (2000)

2. Goggin, K.A., Murphy, D.J.: Monitoring the traceability, safety and authenticity of imported palm oils in Europe. OCL. 25(6), A603 (2018)

3. RASFF. Rapid alert system for food and feed (RASFF) - RASFF portal, 2019 [Accessed 26.02.2019]. Available from: https://ec.europa.eu/food/sites/food/ files/safety/docs/rasff_annual_report_2004_en.pdf

4. Lohumi, S., Lee, S., Lee, H., Cho, B.-K.: A review of vibrational spectroscopic techniques for the detection of authenticity and adulteration. Trends Food Sci. Tech. 46, 85-98 (2015)

5. Rebane, R., Leito, I., Yurchenko, R., Herodes, K.: A review of analytical techniques for determination of Sudan I-IV dyes in food matrixes. J. Chromatogr. A. 1217, 2747-2757 (2010)

6. Larsen, J.C.: Legal and illegal colours. Trends Food Sci. Tech. 19, 64-69 (2008)

7. Brantom, P.G.: Opinion of the scientific panel on food additives, Flavourings, processing aids and materials in contact with food on a request from the commission to review the toxicology of a number of dyes illegally present in food in the EU. EFSA J. 263, 1-71 (2005)

8. Pan, H., Feng, J., He, G.-X., Cerniglia, C.E., Chen, H.: Evaluation of impact of exposure to Sudan azo dyes and their metabolites on human intestinal bacteria. Anaerobe. 18, 445-453 (2012)

9. Pace, N.: Detection of adulterant seed oils in extra virgin olive oils by LC-MS and principal component analysis. AB Sciex. https://sciex.com/Documents/ brochures/olive_oil_analysis3200_1282510.pdf

10. Lv, M.Y., Zhang, X., Ren, H.R., Liu, L., Zhao, Y.M., Wang, Z., Wu, L.Z., Liu, L.M., $\mathrm{Xu}$, H.J.: A rapid method to authenticate vegetable oils through surfaceenhanced Raman scattering. Sci. Rep. 6, 23405 (2016)
11. Gukowsky, J.C., Xie, T., Gao, S., Qu, Y., He, L.: Rapid identification of artificial and natural food colorants with surface enhanced Raman spectroscopy. Food Cont. 92, 267-275 (2018)

12. Gordon, R., Chapman, J., Power, A., Chandra, S., Roberts, J., Cozzolino, D.: Unfrazzled by fizziness: identification of beers using attenuated total reflectance mid-infrared spectroscopy and multivariate analysis. Food Anal. Methods. 11, 2360-2367 (2018)

13. Hu, Y., Feng, S., Gao, F., Li-Chan, E.C.Y., Grant, E., Lu, X.: Detection of melamine in milk using molecularly imprinted polymers-surface enhanced Raman spectroscopy. Food Chem. 176, 123-129 (2015)

14. Abdi, H., Williams, L.J.: Principal component analysis. Comp. Stats. 2, 433-459 (2010)

15. Matikainen, A., Nuutinen, T., Vahimaa, P., Honkanen, S.: A solution to the fabrication and tarnishing problems of surface-enhanced Raman spectroscopy (SERS) fiber probes. Sci. Rep. 5, 1-6 (2015)

16. Ferreira, B.S., de Almeida, C.G., Le Hyaric, M., de Oliveira, V.E., Edwards, H.G.M. , de Oliveira, L.F.C.: Raman spectroscopic investigation of carotenoids in oils from Amazonian products. Spect. Lett. 46(2), 122-127 (2013)

\section{Publisher's Note}

Springer Nature remains neutral with regard to jurisdictional claims in published maps and institutional affiliations.

\section{Submit your manuscript to a SpringerOpen ${ }^{\circ}$ journal and benefit from:}

- Convenient online submission

- Rigorous peer review

- Open access: articles freely available online

High visibility within the field

- Retaining the copyright to your article

Submit your next manuscript at $\boldsymbol{\nabla}$ springeropen.com 